\title{
Functional neuroanatomy underlying the clinical subcategorization of minimally conscious state patients
}

\author{
Marie-Aurélie Bruno $\cdot$ Steve Majerus $\cdot$ Mélanie Boly $\cdot$ Audrey Vanhaudenhuyse $\cdot$ Caroline Schnakers \\ Olivia Gosseries · Pierre Boveroux • Murielle Kirsch • Athena Demertzi · Claire Bernard • \\ Roland Hustinx $\cdot$ Gustave Moonen $\cdot$ Steven Laureys
}

Received: 12 July 2011/Revised: 26 September 2011 / Accepted: 24 October 2011

(C) Springer-Verlag 2011

\begin{abstract}
Patients in a minimally conscious state (MCS) show restricted signs of awareness but are unable to communicate. We assessed cerebral glucose metabolism in MCS patients and tested the hypothesis that this entity can be subcategorized into MCS - (i.e., patients only showing nonreflex behavior such as visual pursuit, localization of noxious stimulation and/or contingent behavior) and MCS+ (i.e., patients showing command following).

Patterns of cerebral glucose metabolism were studied using $\left[{ }^{18} \mathrm{~F}\right]$-fluorodeoxyglucose-PET in 39 healthy volunteers (aged $46 \pm 18$ years) and 27 MCS patients of whom 13 were MCS - (aged $49 \pm 19$ years; 4 traumatic; $21 \pm 23$ months post injury) and 14 MCS + (aged $43 \pm 19$ years; 5 traumatic; $19 \pm 26$ months post injury). Results were thresholded for significance at false discovery rate corrected $p<0.05$.
\end{abstract}

M.-A. Bruno - M. Boly · A. Vanhaudenhuyse - C. Schnakers ·

O. Gosseries · P. Boveroux · M. Kirsch · A. Demertzi ·

G. Moonen · S. Laureys $(\bowtie)$

Coma Science Group, Cyclotron Research Center,

University of Liège, Sart-Tilman B30, 4000 Liège, Belgium

e-mail: steven.laureys@ulg.ac.be

M. Boly

e-mail: mboly@ulg.ac.be

A. Vanhaudenhuyse

e-mail: avanhaudenhuyse@ulg.ac.be

C. Schnakers

e-mail: c.schnakers@ulg.ac.be

O. Gosseries

e-mail: ogosseries@ulg.ac.be

P. Boveroux

e-mail: Pierre.Boveroux@chu.ulg.ac.be

M. Kirsch

e-mail: murielle.kirsch@chu.ulg.ac.be

A. Demertzi

e-mail: a.demertzi@ulg.ac.be
We observed a metabolic impairment in a bilateral subcortical (thalamus and caudate) and cortical (frontotemporo-parietal) network in nontraumatic and traumatic MCS patients. Compared to MCS-, patients in MCS+ showed higher cerebral metabolism in left-sided cortical areas encompassing the language network, premotor, presupplementary motor, and sensorimotor cortices. A functional connectivity study showed that Broca's region was disconnected from the rest of the language network, mesiofrontal and cerebellar areas in MCS - as compared to MCS+ patients.

The proposed subcategorization of MCS based on the presence or absence of command following showed a different functional neuroanatomy. MCS - is characterized by preserved right hemispheric cortical metabolism

G. Moonen

e-mail: g.moonen@chu.ulg.ac.be

M.-A. Bruno - S. Majerus - M. Boly $\cdot$ A. Vanhaudenhuyse

C. Schnakers · O. Gosseries · S. Laureys

Fund for Scientific Research FNRS, Brussels, Belgium

e-mail: ma.bruno@ulg.ac.be

S. Majerus

Research Center for Cognitive and Behavioral Neuroscience,

University of Liège, Liège, Belgium

e-mail: smajerus@ulg.ac.be

M. Boly · G. Moonen · S. Laureys

Department of Neurology, University Hospital of Liège, Liège,

Belgium

C. Bernard · R. Hustinx

Department of Nuclear Medicine, University Hospital of Liège,

Liège, Belgium

e-mail: C.Bernard@chu.ulg.ac.be

R. Hustinx

e-mail: rhustinx@chu.ulg.ac.be 
interpreted as evidence of residual sensory consciousness. MCS+ patients showed preserved metabolism and functional connectivity in language networks arguably reflecting some additional higher order or extended consciousness albeit devoid of clinical verbal or nonverbal expression.

Keywords Coma $\cdot$ Consciousness - Minimally conscious state $\cdot$ Positron-emission tomography $\cdot$ Neuroanatomy

\section{Introduction}

The minimally conscious state (MCS) describes a condition of severely altered consciousness in which patients demonstrate minimal but definite behavioral evidence of awareness but are, by definition, unable to effectively communicate [1]. We recently propose to subcategorize MCS patients based on the complexity of their behavior into two entities: "MCS minus" (MCS-) and "MCS plus" (MCS+) [2]. MCS - describes patients with minimal level of behavioral interactions without command following (e.g., visual pursuit, localization of noxious stimulation and/or smiling/crying in contingent relationship to external stimuli). MCS + patients show higher-level behavioral responses such as command following (Figure 1). Since the subcategorization of MCS is based on the complexity of behavior as previously proposed [2], the aim of this study was to characterize the integrity of residual cortical networks in MCS patients using $\left[{ }^{18} \mathrm{~F}\right]$-fluorodeoxyglucosePET (FDG-PET) testing the hypothesis that this heterogeneous clinical entity can be subcategorized into MCS - and MCS+, each subcategory characterized by its own functional neuroanatomy.

\section{Methods}

Cerebral metabolic rates for glucose (CMRGlu) [3] were studied by means of FDG-PET in 27 subacute and chronic MCS patients (10 women; aged $45 \pm 16$ years) and 39 agematched healthy controls ( 21 women; aged $46 \pm 18$ years). Inclusion criteria were the presence of acute brain damage, coma on admission, absence of sedation, and the presence of operational criteria for MCS (i.e., patients showing minimal but definite behavioral evidence of awareness). Exclusion criteria were patients with extremely vast structural damage encompassing more than two-thirds of one hemisphere in order to allow for reliable spatial normalization of brain images. Table 1 shows the patients' demographic and clinical data. Patients were diagnosed as being in a MCS according to the Aspen Neurobehavioral Conference Workgroup clinical criteria [1] and had repeated Coma Recovery Scale-Revised (CRS-R) [4] assessments (day of scanning, and in the week before and the week after) performed by an experienced multidisciplinary team (i.e., neurologists $\mathrm{MB}$ and $\mathrm{SL}$ and neuropsychologists MAB, AV, $\mathrm{CS}, \mathrm{OG}$, and $\mathrm{AD}$ ). Given the known behavioral variability in this pathology, the diagnosis of MCS - was made if the patient repeatedly failed to show command following on all CRS-R assessments. Thirteen patients were classified as MCS - (6 women; aged $49 \pm 19$ years; 4 traumatic; $21 \pm 23$ months post injury) meaning they did not show command following but presented clearly discernible evidence of nonreflex "purposeful" behavior. Nonreflex behavior included (1) localization to noxious stimulation, (2) visual pursuit movements, (3) "automatic" motor responses (e.g., mouth opening to an approaching spoon, nose scratching, grasping the bedrail), (4) object manipulation (i.e., nongrasp reflex hand movements), (5) affective
Fig. 1 Clinical criteria of disorders of consciousness illustrating the proposed difference between MCS - and $\mathrm{MCS}+$. MCS - describes patients with minimal level of behavioral interactions such as visual pursuit, localization of noxious stimulation and/or appropriate smiling/crying. MCS + is characterized by the presence of high-level behavioral responses as command following

\section{EMERGENCE}

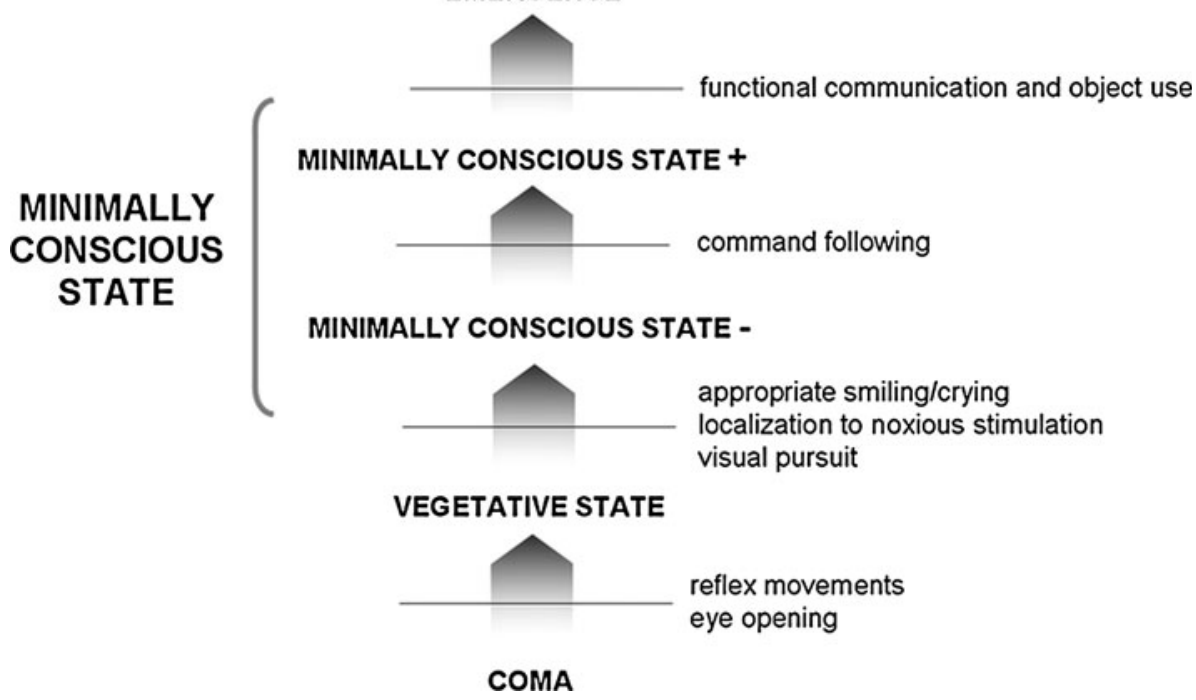




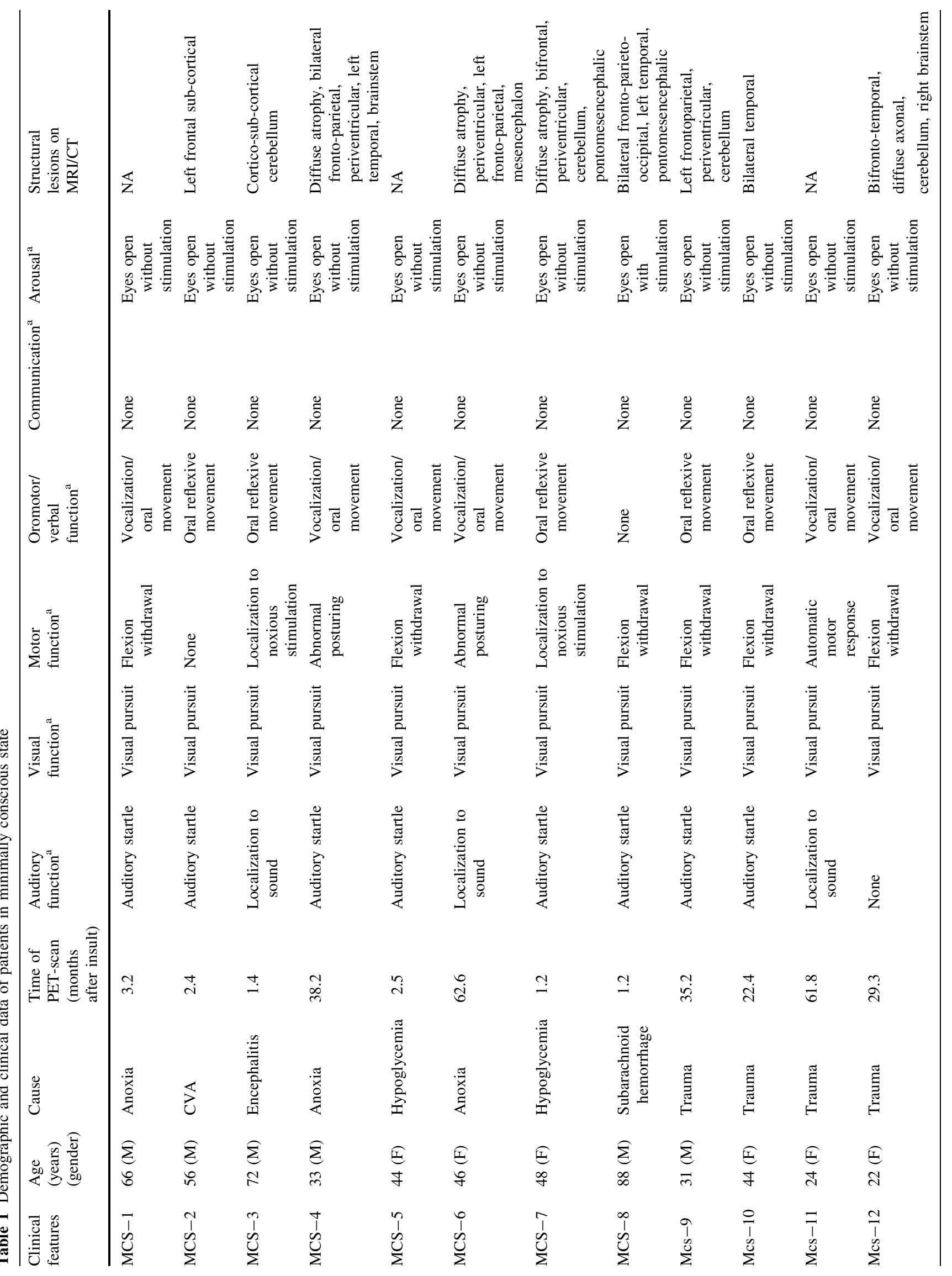




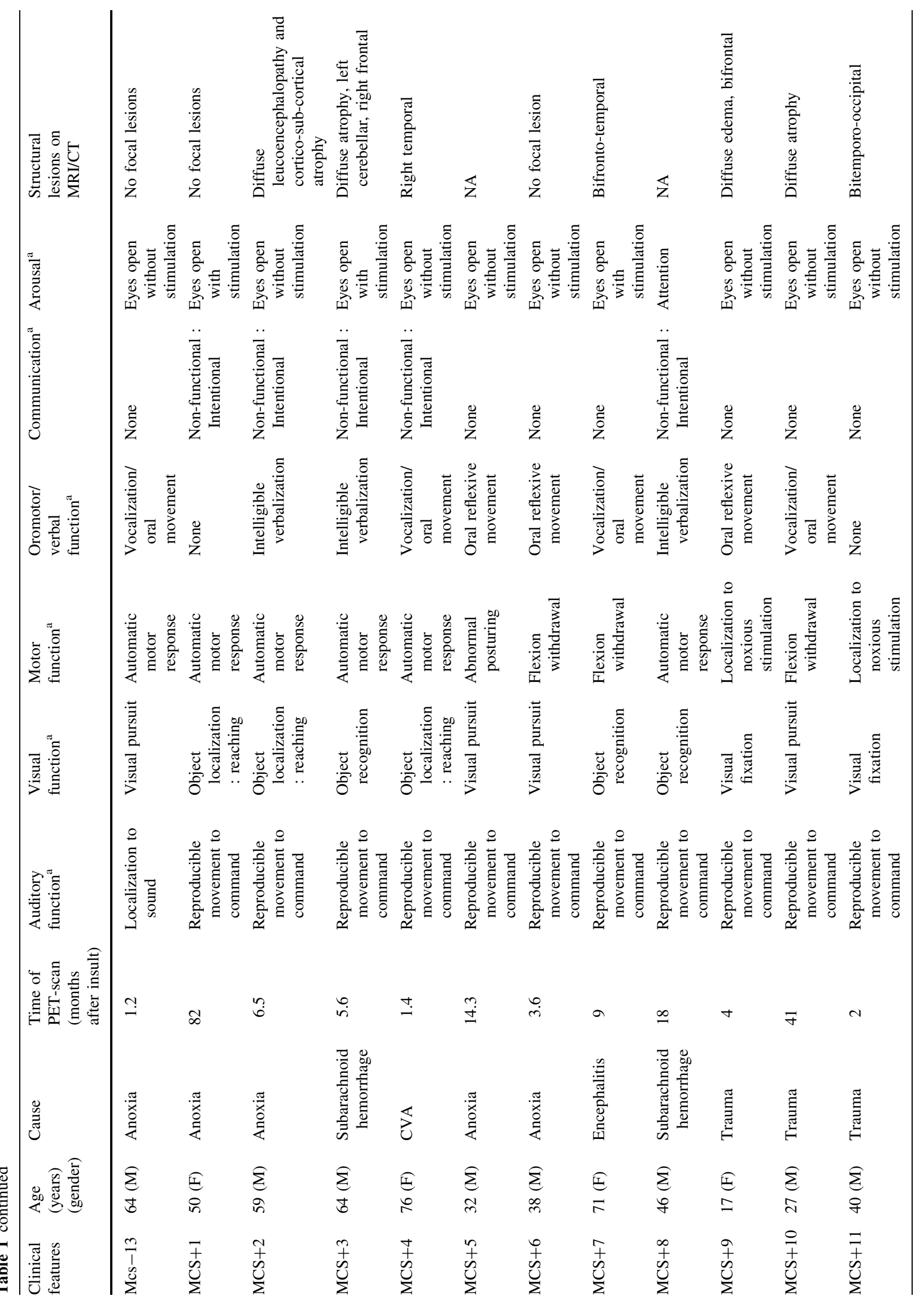




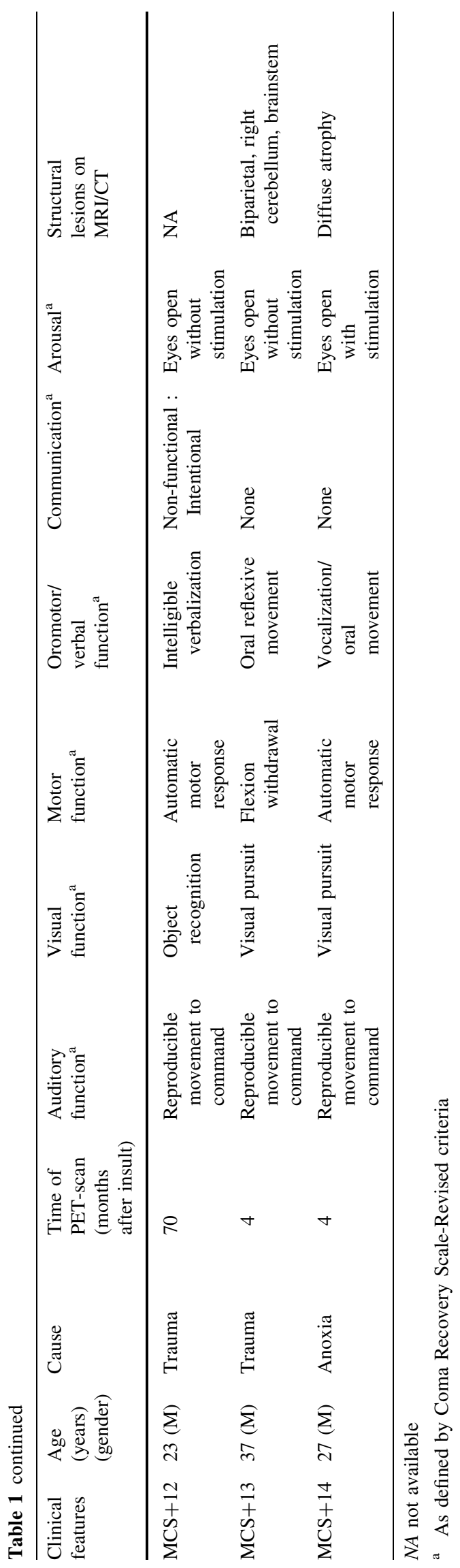

behaviors occurring in contingent relation to relevant environmental stimuli and are not due to reflexive activity (e.g., smiling in response to a specific eliciting stimulus such as the patient's mother) as defined by CRS-R criteria [4]. Fourteen patients were classified as MCS+ (4 women; aged $43 \pm 19$ years; 5 traumatic; $19 \pm 26$ months post injury) meaning they showed reproducible command following (i.e., at least three correct responses out of four identical commands) as defined by CRS-R criteria. Commands were presented verbally and in written form. All patients were studied free of sedative drugs and following administration of a standardized arousal facilitation protocol [4]. MCSand MCS + groups were matched for age, etiology, and time since insult.

FDG-PET data were acquired after intravenous injection of $300 \mathrm{MBq}$ of FDG on a Philips Gemini TF PET-CT scanner [5]. Patients were monitored by two anesthesiologists throughout the procedure. PET data were spatially normalized, smoothed $(14 \mathrm{~mm}$ full width at a half maximum) and analyzed using Statistical Parametric Mapping (SPM8; www.fil.ion.ucl.ac.uk/spm). The first analysis identified brain regions with significant decreased metabolism in the following: (1) MCS patients as compared to controls, (2) MCS - compared to control subjects, (3) MCS + compared to control subjects, (4) MCS + compared to MCS - . The design matrix included 13 MCS-, 14 MCS+, and 39 control subjects' scans and global normalization was performed by proportional scaling. A second analysis looked for differences in brain metabolism between MCS of traumatic $(n=9)$ and nontraumatic $(n=18)$ origin, taking into account age and duration since onset as confounding factors. A third analysis identified brain regions where residual metabolism correlated with the time spent since onset. We also identified areas showing a correlation with the CRS-R total scores. Next, a psychophysiological interaction analysis [3] tested the hypothesis on altered functional cortical connectivity in MCS- as compared to MCS+ and control subjects. This design matrix included the same scans as described above and took into account group differences in mean levels of glucose consumption. Now the analysis looked for brain regions that experienced a significant difference in reciprocal modulation with/from the cortical area that most differentiated MCS - from MCS + (i.e., Broca's area; stereotaxic coordinates $-44,22,4 \mathrm{~mm}$ ). It assessed the difference in modulation of Broca's area depending on the condition MCSversus MCS + or control subjects. A conjunction analysis [6] identified areas showing functional connectivity (i.e., crosscorrelation in metabolic activity) with the seed voxel (Broca's area) in healthy controls which also showed higher connectivity in MCS+ as compared to MCS-.

All results were corrected for multiple comparisons and considered significant at false discovery rate corrected 
Table 2 Statistical results and localization of peak voxels where cerebral metabolism was impaired in patients in a minimally conscious state (MCS), MCS - (showing non-reflex behavior without command following) and MCS + (showing command following)

\begin{tabular}{|c|c|c|c|c|c|c|}
\hline Brain region (area) & Side & $x(\mathrm{~mm})$ & $y(\mathrm{~mm})$ & $z(\mathrm{~mm})$ & $z$-value & Corrected $p$-value \\
\hline \multicolumn{7}{|l|}{ MCS } \\
\hline \multirow[t]{2}{*}{ Caudate } & $\mathrm{L}$ & -8 & 12 & 10 & 6.15 & $<0.001$ \\
\hline & $\mathrm{R}$ & 14 & 14 & 8 & 7.6 & $<0.001$ \\
\hline Bilateral thalamus & & 6 & -16 & 8 & 7.24 & $<0.001$ \\
\hline Posterior cingulate/precuneus (31/7) & & 0 & -30 & 32 & Inf & $<0.001$ \\
\hline Anterior cingulate $(24 / 31)$ & $\mathrm{R}$ & 4 & 12 & 24 & 5.83 & $<0.001$ \\
\hline Premotor (6) & $\mathrm{L}$ & -30 & 6 & 52 & 5.26 & $<0.001$ \\
\hline Middle frontal gyrus (9) & $\mathrm{L}$ & -44 & 12 & 34 & 4.66 & $<0.001$ \\
\hline Superior frontal gyrus (10) & $\mathrm{L}$ & -32 & 54 & 14 & 3.73 & 0.001 \\
\hline Inferior frontal gyrus (47) & $\mathrm{L}$ & -40 & 20 & -4 & 4.69 & $<0.001$ \\
\hline Premotor cortex (6) & $\mathrm{R}$ & 38 & 4 & 50 & 4.50 & $<0.001$ \\
\hline Superior frontal gyrus (8) & $\mathrm{R}$ & 2 & 18 & 50 & 4.64 & $<0.001$ \\
\hline Superior temporal gyrus (38) & $\mathrm{R}$ & 42 & 24 & -22 & 4.5 & $<0.001$ \\
\hline \multirow[t]{2}{*}{ Inferior temporal gyrus (20) } & $\mathrm{L}$ & -62 & -26 & -16 & 2.88 & 0.013 \\
\hline & $\mathrm{R}$ & 64 & -18 & -18 & 2.95 & 0.011 \\
\hline \multirow[t]{2}{*}{ Angular gyrus (39/40) } & $\mathrm{L}$ & -44 & -70 & 42 & 3.68 & 0.001 \\
\hline & $\mathrm{R}$ & 54 & -52 & 52 & 2.99 & 0.010 \\
\hline \multicolumn{7}{|l|}{ MCS- } \\
\hline \multirow[t]{2}{*}{ Caudate } & $\mathrm{L}$ & -8 & 12 & 8 & 5.75 & $<0.001$ \\
\hline & $\mathrm{R}$ & 14 & 14 & 8 & 6.44 & $<0.001$ \\
\hline Bilateral thalamus & & 0 & -18 & 6 & 5.82 & $<0.001$ \\
\hline Posterior cingulate/precuneus $(31 / 7)$ & & 0 & -26 & 32 & 6.93 & $<0.001$ \\
\hline Anterior cingulate (33) & $\mathrm{R}$ & 4 & 12 & 24 & 5.5 & $<0.001$ \\
\hline Premotor $(6)$ & $\mathrm{L}$ & -30 & 8 & 54 & 5.96 & $<0.001$ \\
\hline \multirow[t]{2}{*}{ Middle frontal gyrus $(9 / 6)$} & $\mathrm{L}$ & -44 & 14 & 32 & 5.61 & $<0.001$ \\
\hline & $\mathrm{R}$ & 36 & 4 & 62 & 4.18 & $<0.001$ \\
\hline Inferior frontal gyrus (47) & $\mathrm{L}$ & -40 & 20 & -2 & 5.49 & $<0.001$ \\
\hline \multirow[t]{2}{*}{ Inferior temporal gyrus (20) } & $\mathrm{L}$ & -60 & -22 & -16 & 3.96 & 0.001 \\
\hline & $\mathrm{R}$ & 62 & -20 & -18 & 2.42 & 0.008 \\
\hline \multirow[t]{2}{*}{ Angular gyrus (39/38) } & $\mathrm{L}$ & -46 & -68 & 40 & 4.58 & $<0.001$ \\
\hline & $\mathrm{R}$ & 42 & 24 & -22 & 3.18 & 0.006 \\
\hline \multicolumn{7}{|l|}{ MCS+ } \\
\hline \multirow[t]{2}{*}{ Caudate } & $\mathrm{L}$ & -8 & 10 & 12 & 4.48 & $<0.001$ \\
\hline & $\mathrm{R}$ & 14 & 14 & 6 & 6.53 & $<0.001$ \\
\hline Right thalamus & $\mathrm{R}$ & 10 & -16 & 10 & 6.86 & $<0.001$ \\
\hline Posterior cingulate/precuneus (31/7) & & 0 & -36 & 32 & 7.04 & $<0.001$ \\
\hline Anterior cingulate (32) & $\mathrm{R}$ & 8 & 12 & 38 & 4.21 & $<0.001$ \\
\hline Middle frontal gyrus (9) & $\mathrm{R}$ & 46 & 12 & 30 & 4.3 & $<0.001$ \\
\hline Middle temporal gyrus (21) & $\mathrm{R}$ & 68 & -50 & -2 & 2.67 & 0.033 \\
\hline Superior temporal gyrus (22) & $\mathrm{R}$ & 52 & 12 & -4 & 4.89 & $<0.001$ \\
\hline Postcentral gyrus (7) & $\mathrm{R}$ & 38 & -70 & 52 & 2.69 & 0.031 \\
\hline Angular gyrus (39/40) & $\mathrm{R}$ & 54 & -50 & 52 & 3.08 & 0.012 \\
\hline Right premotor gyrus & $\mathrm{R}$ & 48 & 10 & 48 & 3.85 & $<0.001$ \\
\hline \multicolumn{7}{|c|}{ Preserved area in MCS+ as compared to MCS- } \\
\hline Caudate & $\mathrm{L}$ & -8 & 8 & -6 & 2.62 & 0.048 \\
\hline Sensory-motor area $(4 / 3)$ & $\mathrm{L}$ & -60 & -8 & 26 & 3.37 & 0.023 \\
\hline Premotor (6) & $\mathrm{L}$ & -30 & 8 & 56 & 3.76 & 0.023 \\
\hline Inferior frontal gyrus (45) & $\mathrm{L}$ & -44 & 22 & 4 & 3.99 & 0.023 \\
\hline
\end{tabular}


Table 2 continued

\begin{tabular}{lllcrrr}
\hline Brain region (area) & Side & $x(\mathrm{~mm})$ & $y(\mathrm{~mm})$ & $z(\mathrm{~mm})$ & $z$-value & Corrected $p$-value \\
\hline Middle frontal gyrus (9) & $\mathrm{L}$ & -42 & 14 & 30 & 3.87 & 0.023 \\
Superior temporal gyrus (39) & $\mathrm{L}$ & -58 & -56 & 26 & 3.19 & 0.024 \\
Middle temporal gyrus (21) & $\mathrm{L}$ & -54 & -8 & -14 & 3.77 & 0.023 \\
\hline
\end{tabular}

Coordinates are in standardized stereotaxic Montreal Neurological Institute space

Fig. 2 Areas with impaired metabolism (shown in blue) in patients in a minimally conscious state (MCS), MCS(showing nonreflex behavior) and MCS + (showing command following). The lowest panel shows areas with higher metabolism in MCS+ as compared to MCS - (shown in orange). All results are shown on a 3D MRI template and thresholded at false discovery rate corrected $p<0.05$

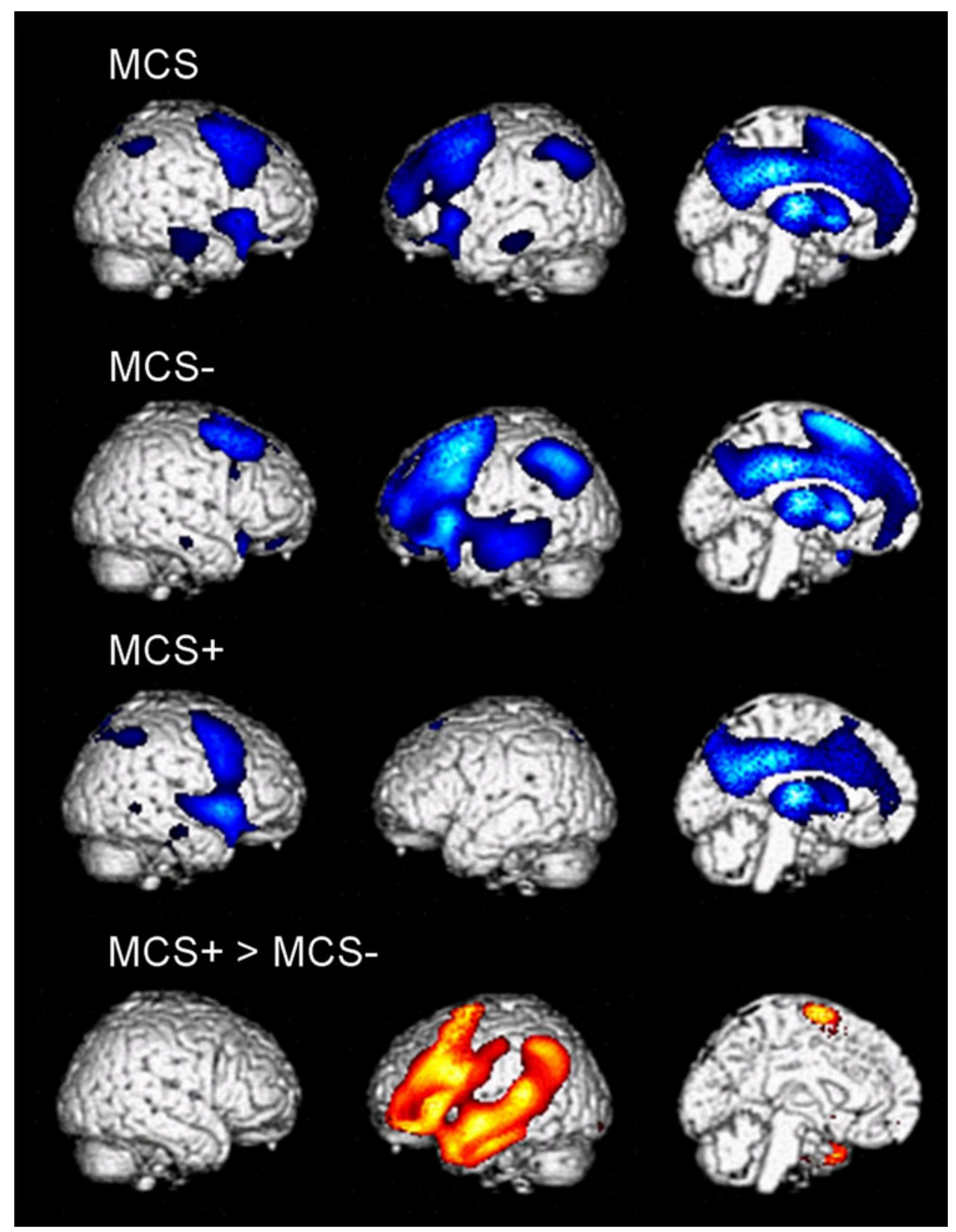

\section{Results}

Patients in MCS (as compared to healthy controls) showed hypometabolism in bilateral thalamus, caudate, posterior $p<0.05$ [7]. The study was approved by the Ethics Committee of the Faculty of Medicine of the University of Liege and written informed consent was obtained from all healthy controls and the patients' legal representatives. 
Table 3 Peak voxels showing linear correlation between regional metabolism and Coma Recovery Scale-Revised total scores

\begin{tabular}{|c|c|c|c|c|c|c|}
\hline Brain region (area) & Side & $x(\mathrm{~mm})$ & $y(\mathrm{~mm})$ & $z(\mathrm{~mm})$ & $z$-value & $\begin{array}{l}\text { Corrected } \\
p \text {-value }\end{array}$ \\
\hline \multirow[t]{2}{*}{ Left caudate } & $\mathrm{L}$ & -10 & 12 & 8 & 6.60 & $<0.001$ \\
\hline & $\mathrm{R}$ & 14 & 14 & 8 & 6.63 & $<0.001$ \\
\hline Left thalamus & $\mathrm{L}$ & -2 & -18 & 6 & 6.76 & $<0.001$ \\
\hline \multirow[t]{2}{*}{ Insula } & $\mathrm{L}$ & -38 & 20 & -4 & 5.59 & $<0.001$ \\
\hline & $\mathrm{R}$ & 38 & 24 & -2 & 3.33 & $<0.001$ \\
\hline Posterior cingulate (31) & & 0 & -28 & 34 & 7.71 & $<0.001$ \\
\hline Precuneus (7) & $\mathrm{L}$ & -2 & -70 & 38 & 4.27 & $<0.001$ \\
\hline Anterior cingulate (33) & $\mathrm{R}$ & 4 & 12 & 24 & 5.82 & $<0.001$ \\
\hline Mesiofrontal (10) & & 0 & 52 & 0 & 3.46 & 0.002 \\
\hline \multirow[t]{2}{*}{ Premotor (6) } & $\mathrm{L}$ & -30 & 8 & 52 & 6.21 & $<0.001$ \\
\hline & $\mathrm{R}$ & 34 & 6 & 64 & 4.09 & $<0.001$ \\
\hline \multirow[t]{2}{*}{ Superior frontal gyrus (8) } & $\mathrm{L}$ & -4 & 48 & 42 & 4.31 & $<0.001$ \\
\hline & $\mathrm{R}$ & 26 & 28 & 54 & 3.76 & 0.001 \\
\hline \multirow[t]{2}{*}{ Middle frontal gyrus $(9 / 10)$} & $\mathrm{L}$ & -44 & 14 & 30 & 5.92 & $<0.001$ \\
\hline & $\mathrm{L}$ & -36 & 52 & 12 & 4.70 & $<0.001$ \\
\hline Inferior parietal gyrus (39) & $\mathrm{L}$ & -46 & -68 & 42 & 5.01 & $<0.001$ \\
\hline $\begin{array}{l}\text { Middle temporal gyrus (21)/ } \\
\text { Primary auditory area }\end{array}$ & $\mathrm{L}$ & -62 & -26 & -14 & 3.68 & 0.001 \\
\hline
\end{tabular}

cingulate and precuneal, anterior cingulate and mesiofrontal, posterior parietal, temporal, and dorsolateral prefrontal cortices. Patients in MCS - showed metabolic dysfunction in bilateral thalami, caudate, posterior cingulate and precuneal, anterior cingulate and mesiofrontal, angular gyrus, left posterior parietal and bilateral temporal and dorsolateral prefrontal cortices. Patients in MCS + showed hypometabolism in right thalamus, bilateral caudate, posterior cingulate and precuneal, anterior cingulate and mesiofrontal, right posterior parietal, temporal, and premotor cortices. Finally, MCS+ patients showed significant higher metabolism as compared to MCS - in Broca's and Wernicke's regions, left premotor, left caudate, and post- and precentral cortices (Table 2; Fig. 2). No differences were observed between traumatic and nontraumatic etiology.

This analysis as a function of lesion type was also performed for each MCS group separately (MCS - traumatic versus $\mathrm{MCS}$ - nontraumatic; MCS+ traumatic versus $\mathrm{MCS}+$ nontraumatic). No differences were found between traumatic and nontraumatic groups within each subcategory. Time since onset showed no negative correlation with brain metabolism (similar results were observed when acute ( $<3$ months) and subacute/chronic patients were compared). Conversely, a positive correlation was observed with precuneus-albeit at a less conservative threshold (coordinates $x=10 y=-58 \quad z=48 \mathrm{~mm}$, $z$ value $=3.72$; small volume corrected $p<0.05$ ).

CRS-R total scores showed a linear correlation with metabolism in the left thalamus, precuneus, posterior parietal, left primary and associative auditory cortices, bilateral premotor cortex, frontal eye field, insula, dorsolateral prefrontal and anterior cingulate cortices (Table 3; Fig. 3).

The connectivity study showed that Broca's area was functionally connected with the language network, mesiofrontal and cerebellar areas in controls and that this connectivity was significantly higher in $\mathrm{MCS}+$ as compared to MCS- patients (Table 4; Fig. 4).

\section{Discussion}

Metabolic impairment in a bilateral subcortical (thalamus and caudate) and cortical (fronto-temporo-parietal) network in nontraumatic and traumatic MCS patients, comparable but less widespread than previously shown in the vegetative state [3], was shown with this research. These results are in line and extend a previous FDG-PET study in 13 MCS patients of traumatic origin [8]. We observed no differences in residual brain function depending on etiology of MCS. In the vegetative state, a progressive loss of cortical metabolic function was reported as a function of the duration of the condition. The absence of such decreased brain metabolism with time in our MCS cohort might illustrate the absence of progressive Wallerian and transsynaptic degeneration characterizing the chronic vegetative state [9]. In contrast, an increase in metabolism seemed present in some areas such as the precuneus in our subacute and chronic MCS patients. This can be interpreted as a sign of residual cortical plasticity in areas previously identified by means of diffusion tensor 

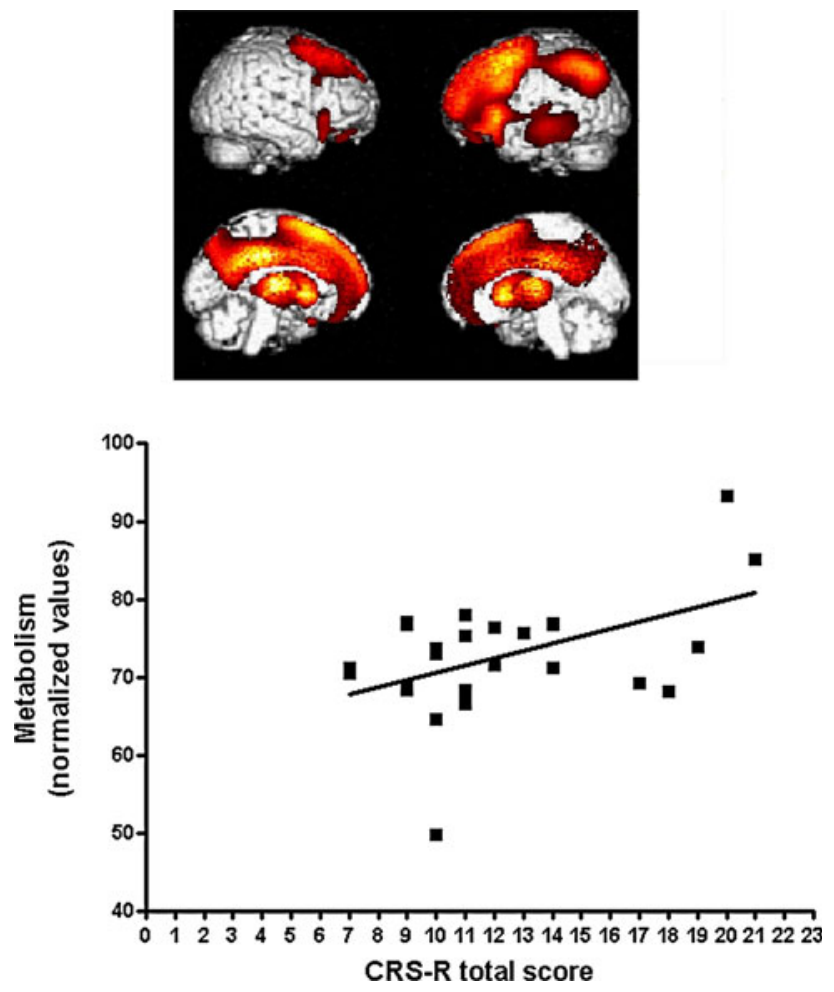

Fig. 3 Areas showing linear correlation of metabolism with Coma Recovery Scale-Revised (CRS-R) total scores (shown in red) shown on a 3D MRI template and thresholded at false discovery rate corrected $p<0.05$. The lower panel graphically illustrates that CRS$\mathrm{R}$ scores increase as metabolic activity becomes more robust

MRI techniques in exceptional cases of recovery from longstanding MCS [10].

We found a positive linear correlation between metabolism in frontoparietal cortices and the CRS-R total scores, in line with our previous study showing a correlation between this behavioral score and spontaneous "default network" brain activity as measured by functional MRI in "resting state" conditions [11]. The identified polymodal frontoparietal network is considered critical for the emergence of conscious awareness [12]. These results corroborate previous findings on pain $[13,14]$, auditory $[15,16]$,

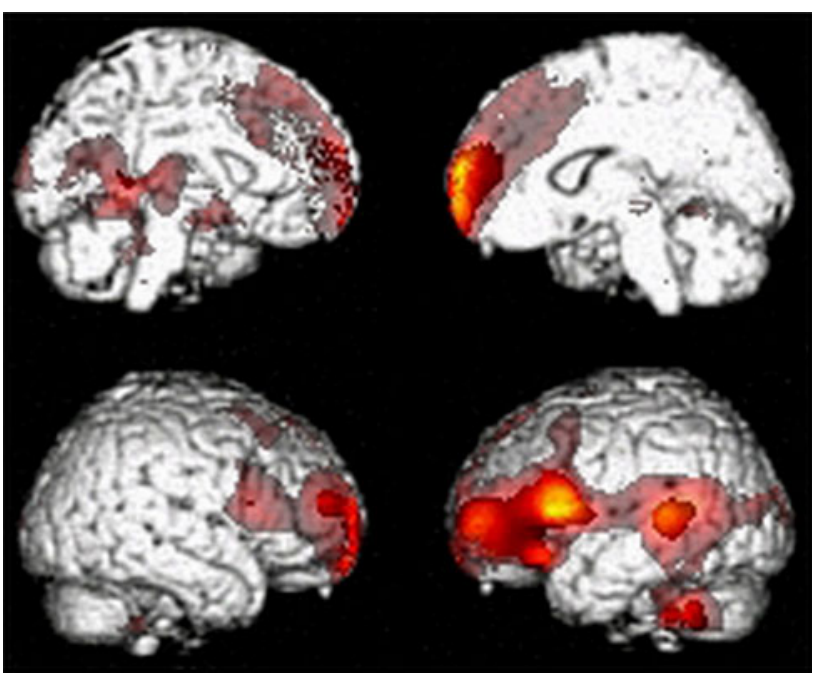

Fig. 4 Functional disconnections with Broca's area in MCS- as compared to MCS + showing the language network, mesiofrontal and cerebellar areas (thresholded at false discovery rate corrected $p<0.05$; transparency denotes uncorrected $p<0.05$ )

and emotional $[17,18]$ processing showing that MCS patients demonstrate a more elaborated and integrated level of noxious, auditory, and emotional processing than vegetative state patients who only showed activation of primary "lower level" sensory cortices which are disconnected from "higher order" associative cortical networks [5, 14].

Clinically, the MCS entity regroups patients with different degrees of cognitive dysfunction or disability. We here propose to subcategorize MCS in patients showing only minimal levels of nonreflex behavioral responses (coined MCS-) and patients showing higher levels of behavioral interactions such as command following $(\mathrm{MCS}+)$. Our FDG-PET results showed a different functional neuroanatomy for both groups. MCS - seems characterized by a partially preserved functioning brainstem and right hemisphere with impaired left cortical networks encompassing Broca's and Wernicke's regions, posterior

Table 4 Peak voxels of functional connectivity assessment with Broca's area (peak voxel identified in MCS + > MCS - comparison) identifying areas with higher connectivity in MCS + as compared to MCS - patients

\begin{tabular}{llccccc}
\hline Brain region (area) & Side & $x(\mathrm{~mm})$ & $y(\mathrm{~mm})$ & $z(\mathrm{~mm})$ & $z$-value & Corrected $p$-value \\
\hline Posterior cingulate (30) & $\mathrm{L}$ & -14 & -46 & 6 & 3.37 & 0.029 \\
Middle frontal gyrus (10) & $\mathrm{L}$ & -42 & 52 & 6 & 4.46 & 0.003 \\
Mesiofrontal (10) & $\mathrm{R}$ & 8 & 64 & 0 & 4.77 & 0.001 \\
Superior frontal gyrus (10) & $\mathrm{R}$ & 30 & 54 & 16 & 3.49 & 0.023 \\
Inferior frontal gyrus (44) & $\mathrm{R}$ & 64 & 14 & 6 & 3.09 & 0.048 \\
Middle temporal gyrus (22) & $\mathrm{L}$ & -60 & -40 & -46 & 3.56 & 0.020 \\
Cerebellum & $\mathrm{L}$ & -52 & -54 & 4.15 & 0.005 \\
\hline
\end{tabular}


parietal, presupplementary motor, sensorimotor and premotor cortices. Phylogenetically, the midbrain is capable of driving the eyes to track objects $[19,20]$, and the human phenomenon of blindsight in the absence of occipital lobe function suggests that tracking could be coordinated in the optic tectum [21]. Command following requires a series of cognitive and motor skills, including language comprehension, memory, volition, and motor execution, each depending on the functional integrity of multiple neuronal networks. Given that the vast majority of the patients sample had nonlateralizing injury etiologies, our observation that the syndrome lateralizes points to the critical role of the dominant hemisphere and language functions. These results are in line with a recent fMRI study in disorders of consciousness and locked-in patients suggesting that activity of the language network may serve as an indicator of high-level cognition and possibly volitional processes that cannot be discerned through conventional behavioral assessment alone [22]. Understanding spoken language requires a complex series of processing stages to translate speech sounds into meaning encompassing left-lateralized frontal and temporal cortical regions [23-25] shown to be dysfunctional in MCS-. Moreover, our functional connectivity analysis identified corticocortical disconnections within these networks and with speech motor production networks also involving the cerebellum [26, 27]. This functional connectivity analysis was also performed on patients without left focal lesions and showed the same results. Previous work suggested that complementary analyses should be used in studies comprising of traumatic patients. Some of these patients may exhibit extreme focal lesions and, as a result, any statistical inference could be driven by such outliers [28]. Previous studies have located various subprocesses of verbal working memory in structures of the left inferior frontal gyrus [29]. The capacity for voluntary action is thought to depend on the functional integrity of presupplementary motor area, anterior prefrontal, and parietal cortices [30]. Finally, limb praxis control and motor sequencing are considered to depend mainly upon left frontoparietal circuits [31]. All the aforementioned areas were observed to be highly dysfunctional in MCS - but showed near-normal metabolism in MCS+ patients characterized by the clinical demonstration of reproducible but inconsistent command following.

Our findings on MCS - suggest that the metabolically functionally preserved but isolated right hemisphere function might permit these patients to show nonreflex behaviors such as visual pursuit, localization to pain, "automatic" movements such as scratching or affective behaviors contingent upon emotionally relevant stimuli [32], while remaining unable to show command following as an unambiguous clinical proof of consciousness [31,
33]. Split-brain research has previously identified different cognitive processing styles for each cerebral hemisphere [34]. The right hemisphere appears to process what it perceives and no more, while the left hemisphere is considered to make elaborations above the level of minimal sensory consciousness [35]. It should be noted that MCS - patients also showed more frequently left-sided damage on structural MRI scans (5 out of 10 patients), while MCS+ patients had most frequently right hemispheric lesions (3 out of 10 patients). Structural and metabolic functions are obviously linked. These findings can also be seen in light of Damasio's [36] prelinguistic core consciousness seemingly present in both MCS - and MCS + patients. Core consciousness corresponds to the transient process that is incessantly generated relative to any external stimulation without requiring language. Similarly, Edelman has differentiated "primary" from "higher order" consciousness, the latter considered to require language for its most developed expression [37]. Damasio's extended consciousness is a more complex process depending on an autobiographical self and is enhanced by language, possibly partially preserved in MCS+. However, both MCS - and MCS+ patients showed a functional impairment in midline cortices (mesiofrontal and precuneus) considered critical for the emergence of self consciousness [38]. Our limited understanding of the dynamical neural complexity underlying consciousness and its resistance to quantification in the absence of communication [39] makes it difficult to establish strong claims about the selfconsciousness in MCS patients. In our view, even MCS patients lacking clinical proof of consciousness in terms of command following (here coined MCS-) show a functional neuroanatomy reflecting the presence of preserved sensory, core, or primary consciousness. An alternative explanation might be that MCS - patients have a comparable level of consciousness as MCS+ patients but that they fail to understand verbal or written commands due to a selective impairment of language function [40]. This may also explain why some MCS patients fail to show activations during fMRI active paradigms [22, 41]. As discussed elsewhere since language function may impact CRS-R scores, detecting impaired language networks may represent an important factor to consider for the clinical evaluation of patients with disorders of consciousness. This result emphasizes the importance of language independent assessment [42].

Acknowledgments The funding sources had no role in the study design, data collection, data analysis, data interpretation, or writing of this report. All authors had full access to all the data in the study and had final responsibility for the decision to submit for publication. This research was funded by the Belgian National Funds for Scientific Research (FNRS), the European Commission (Mindbridge, DISCOS, 
Marie-Curie Actions, DECODER \& COST), the James McDonnell Foundation, the Mind Science Foundation, the French Speaking Community Concerted Research Action (ARC-06/11-340), the Fondation Médicale Reine Elisabeth, and the University of Liège. SL and MAB participated in the conception and design of this study. MAB, $\mathrm{AV}, \mathrm{CS}, \mathrm{OG}, \mathrm{PB}$, and $\mathrm{MK}$ acquired the data. SL, MAB, and MB analyzed and interpreted the data. MAB and SL drafted the manuscript. AV, CS, AD, OG, MS, GM, and RH revised the manuscript for intellectual content. SL and GM obtained funding. RH and GM provided administrative, technical, or material support, and SL supervised the study.

Conflict of interest The authors declare that they have no competing interests.

\section{References}

1. Giacino JT, Ashwal S, Childs N et al (2002) The minimally conscious state: definition and diagnostic criteria. Neurology 58:349-353

2. Bruno MA, Vanhaudenhuyse A, Thibaut A et al (2011) From unresponsive wakefulness to minimally conscious PLUS and functional locked-in syndromes: recent advances in our understanding of disorders of consciousness. J Neurol

3. Laureys S, Goldman S, Phillips C et al (1999) Impaired effective cortical connectivity in vegetative state: preliminary investigation using PET. Neuroimage 9:377-382

4. Giacino JT, Kalmar K, Whyte J (2004) The JFK Coma Recovery Scale-Revised: measurement characteristics and diagnostic utility. Arch Phys Med Rehabil 85:2020-2029

5. Laureys S, Faymonville ME, Degueldre C et al (2000) Auditory processing in the vegetative state. Brain 123:1589-1601

6. Friston KJ, Penny WD, Glaser DE (2005) Conjunction revisited. Neuroimage 25:661-667

7. Genovese CR, Lazar NA, Nichols T (2002) Thresholding of statistical maps in functional neuroimaging using the false discovery rate. Neuroimage 15:870-878

8. Nakayama N, Okumura A, Shinoda J et al (2006) Relationship between regional cerebral metabolism and consciousness disturbance in traumatic diffuse brain injury without large focal lesions: an FDG-PET study with statistical parametric mapping analysis. J Neurol Neurosurg Psychiatr 77:856-862

9. Rudolf J, Ghaemi M, Ghaemi M et al (1999) Cerebral glucose metabolism in acute and persistent vegetative state. J Neurosurg Anesthesiol 11:17-24

10. Voss HU, Uluc AM, Dyke JP et al (2006) Possible axonal regrowth in late recovery from the minimally conscious state. J Clin Invest 116:2005-2011

11. Vanhaudenhuyse A, Noirhomme Q, Tshibanda LJ et al (2010) Default network connectivity reflects the level of consciousness in non-communicative brain-damaged patients. Brain 133:161-171

12. Laureys S (2005) The neural correlate of (un)awareness: lessons from the vegetative state. Trends Cogn Sci 9:556-559

13. Boly M, Faymonville ME, Schnakers C et al (2008) Perception of pain in the minimally conscious state with PET activation: an observational study. Lancet Neurol 7:1013-1020

14. Laureys S, Faymonville ME, Peigneux P et al (2002) Cortical processing of noxious somatosensory stimuli in the persistent vegetative state. Neuroimage 17:732-741

15. Boly M, Faymonville ME, Peigneux P et al (2004) Auditory processing in severely brain injured patients: differences between the minimally conscious state and the persistent vegetative state. Arch Neurol 61:233-238

16. Coleman MR, Rodd JM, Davis MH et al (2007) Do vegetative patients retain aspects of language comprehension? Evidence from fMRI. Brain 130:2494-2507

17. Laureys S, Perrin F, Faymonville ME et al (2004) Cerebral processing in the minimally conscious state. Neurology 63:916-918

18. Schiff ND, Rodriguez-Moreno D, Kamal A et al (2005) fMRI reveals large-scale network activation in minimally conscious patients. Neurology 64:514-523

19. Bruno MA, Vanhaudenhuyse A, Schnakers C et al (2010) Visual fixation in the vegetative state: an observational case series PET study. BMC Neurol 10:35

20. Kentridge RW, Nijboer TC, Heywood CA (2008) Attended but unseen: visual attention is not sufficient for visual awareness. Neuropsychologia 46:864-869

21. Tamietto M, Cauda F, Corazzini LL et al (2009) Collicular vision guides nonconscious behavior. J Cogn Neurosci 22:888-902

22. Moreno DR, Schiff ND, Giacino J et al (2010) A network approach to assessing cognition in disorders of consciousness. Neurology 75:1871-1878

23. Longoni F, Grande M, Hendrich V et al (2005) An fMRI study on conceptual, grammatical, and morpho-phonological processing. Brain Cogn 57:131-134

24. Vigneau M, Beaucousin V, Herve PY et al (2006) Meta-analyzing left hemisphere language areas: phonology, semantics, and sentence processing. Neuroimage 30:1414-1432

25. Davis MH, Johnsrude IS (2003) Hierarchical processing in spoken language comprehension. J Neurosci 23:3423-3431

26. Hickok G, Poeppel D (2007) The cortical organization of speech processing. Nat Rev Neurosci 8:393-402

27. Riecker A, Mathiak K, Wildgruber D et al (2005) fMRI reveals two distinct cerebral networks subserving speech motor control. Neurology 64:700-706

28. Zhang J, Mitsis EM, Chu K, Newmark RE et al (2010) Statistical parametric mapping and cluster counting analysis of [18F] FDGPET imaging in traumatic brain injury. $J$ Neurotrauma 27(1):35-49

29. Gabrieli JD, Poldrack RA, Desmond JE (1998) The role of left prefrontal cortex in language and memory. Proc Natl Acad Sci USA. 95:906-913

30. Haggard P (2008) Human volition: towards a neuroscience of will. Nat Rev Neurosci 9:934-946

31. Haaland KY, Elsinger CL, Mayer AR et al (2004) Motor sequence complexity and performing hand produce differential patterns of hemispheric lateralization. J Cogn Neurosci 16:621-636

32. Gainotti G (2001) Disorders of emotional behaviour. J Neurol 248:743-749

33. Rushworth MF, Johansen-Berg H, Gobel SM et al (2003) The left parietal and premotor cortices: motor attention and selection. Neuroimage 20(Suppl 1):S89-S100

34. Gazzaniga MS (2000) Cerebral specialization and interhemispheric communication: does the corpus callosum enable the human condition? Brain 123(Pt 7):1293-1326

35. Turk DJ, Heatherton TF, Macrae CN et al (2003) Out of contact, out of mind: the distributed nature of the self. Ann NY Acad Sci 1001:65-78

36. Damasio AR (1998) Investigating the biology of consciousness. Philos Trans R Soc Lond B Biol Sci 353:1879-1882

37. Edelman GM (2004) Wider than the sky: The phenomenal gift of consciousness. Yale University Press, New Haven and London

38. Laureys S, Perrin F, Bredart S (2007) Self-consciousness in noncommunicative patients. Conscious Cogn 16:722-741 (discussion $742-745)$ 
39. Seth AK, Dienes Z, Cleeremans A et al (2008) Measuring consciousness: relating behavioural and neurophysiological approaches. Trends Cogn Sci 12:314-321

40. Majerus S, Bruno MA, Schnakers C et al (2009) The problem of aphasia in the assessment of consciousness in brain-damaged patients. Prog Brain Res 177:49-61
41. Monti MM, Vanhaudenhuyse A, Coleman MR et al (2010) Willful modulation of brain activity in disorders of consciousness. N. Engl J Med 362(7):579-589

42. Boly M, Garrido MA, Gosseries O et al (2011) Preserved feedforward but impaired top-down processes in the vegetative state. Science 332:858-862 